\title{
Primary intraosseous meningioma: clinical, histological, and differential diagnostic aspects
}

\author{
Sebastian Butscheidt, MD, ${ }^{1,2}$ Marielle Ernst, MD, ${ }^{3}$ Tim Rolvien, MD, PhD, ${ }^{1,2}$ Jan Hubert, MD, ${ }^{1,2}$ \\ Jozef Zustin, MD, ${ }^{4}$ Michael Amling, MD, ${ }^{1}$ and Tobias Martens, MD ${ }^{5}$ \\ Departments of ${ }^{1}$ Osteology and Biomechanics, ${ }^{2}$ Orthopedics, and ${ }^{3}$ Neuroradiological Diagnostics and Intervention, University \\ Medical Center Hamburg-Eppendorf; ${ }^{4}$ Pathology Practice, Pathologie-Hamburg, Lademannbogen; and ${ }^{5}$ Department of \\ Neurosurgery, University Medical Center Hamburg-Eppendorf, Hamburg, Germany
}

\begin{abstract}
OBJECTIVE Primary intraosseous meningioma (PIM) is a rare manifestation of meningioma, a benign, neoplastic lesion of the meninges. Its characteristic appearance is hyperostosis, while no or only minimal dural changes can be observed. This study aims to characterize this rare entity from both a clinical and histopathological point of view in order to improve clinical management.

METHODS In the years 2009-2017, 26 cases of PIM were diagnosed using MRI and CT scans. In 16 cases the indication for resection was given, and specimens were further examined using a multilevel approach, including histological and immunohistochemical analyses. Additionally, the local database was searched for all cases of meningiomas, as well as osteosclerotic differential diagnoses-i.e., fibrous dysplasia, Paget's disease of bone, and other benign osteosclerotic lesions.
\end{abstract}

RESULTS In this study, PIM represented $2.4 \%$ of all meningiomas with a predominant occurrence in females (85\%). Regarding the initial manifestation, PIMs show a slightly earlier onset than meningiomas. While most PIMs are located in the sphenoid bone, associated calcifications were visible in $58 \%$ of the cases on CT scans. Most of the cases were classified as WHO grade I (93\%) and meningotheliomatous meningiomas (91\%). Tumor growth was associated with an increased bone resorption followed by massive osteoid deposition and consecutive sclerosis. The frequently observed frayed appearance results from multiple bony canals, which contain blood vessels for the blood supply of the highly vascularized tumor tissue.

CONCLUSIONS PIM is a rare but important differential diagnosis for osteosclerotic lesions of the skull, especially in women. Tumor-induced, cellular-mediated bone resorption and formation may play a central role in the underlying pathogenesis.

https://thejns.org/doi/abs/10.3171/2019.3.JNS182968

KEYWORDS primary intraosseous meningioma; hyperostosis; pathogenesis; differential diagnoses; treatment; histology; oncology

$\mathrm{M}$ ENINGIOMA is a benign, neoplastic lesion most often originating from arachnoid cells. It represents the most common tumor of the skull, with an estimated incidence of 2.3/100,000. ${ }^{23}$ Neurological symptoms such as headache and visual loss mainly depend on the location of the tumor and usually lead to a radiological evaluation and subsequent presentation of en plaque or en masse manifestations. However, in rare cases, the tumor cells primarily spread within the cranial bone, accompanied by hyperostosis, and the lesions are then classified as primary intraosseous meningiomas (PIMs). Several case reports ${ }^{1,18,26-28}$ and studies ${ }^{3,6,13}$ are available in the literature, but they are incomplete in terms of epidemiological and clinical data, as well as adequate bone analyses. The predominant osseous lesion with only minimal or complete absence of dural involvement makes establishing the correct diagnosis challenging. Relevant differential diagnoses such as fibrous dysplasia (FD), Paget's disease of bone (PDB) of the skull, and benign osteosclerotic lesions (e.g., osteoma) need to be considered. ${ }^{8}$ An improved understanding of PIMs and comparison with differential diagnoses can improve the time to diagnosis and application of appropriate treatment.

This study aims to characterize this rare entity from

ABBREVIATIONS FD = fibrous dysplasia; $\mathrm{PDB}=$ Paget's disease of bone; $\mathrm{PIM}=$ primary intraosseous meningioma; $q \mathrm{BEI}=$ quantitative backscattered electron imaging . SUBMITTED October 19, 2018. ACCEPTED March 29, 2019.

INCLUDE WHEN CITING Published online June 21, 2019; DOI: 10.3171/2019.3.JNS182968. 

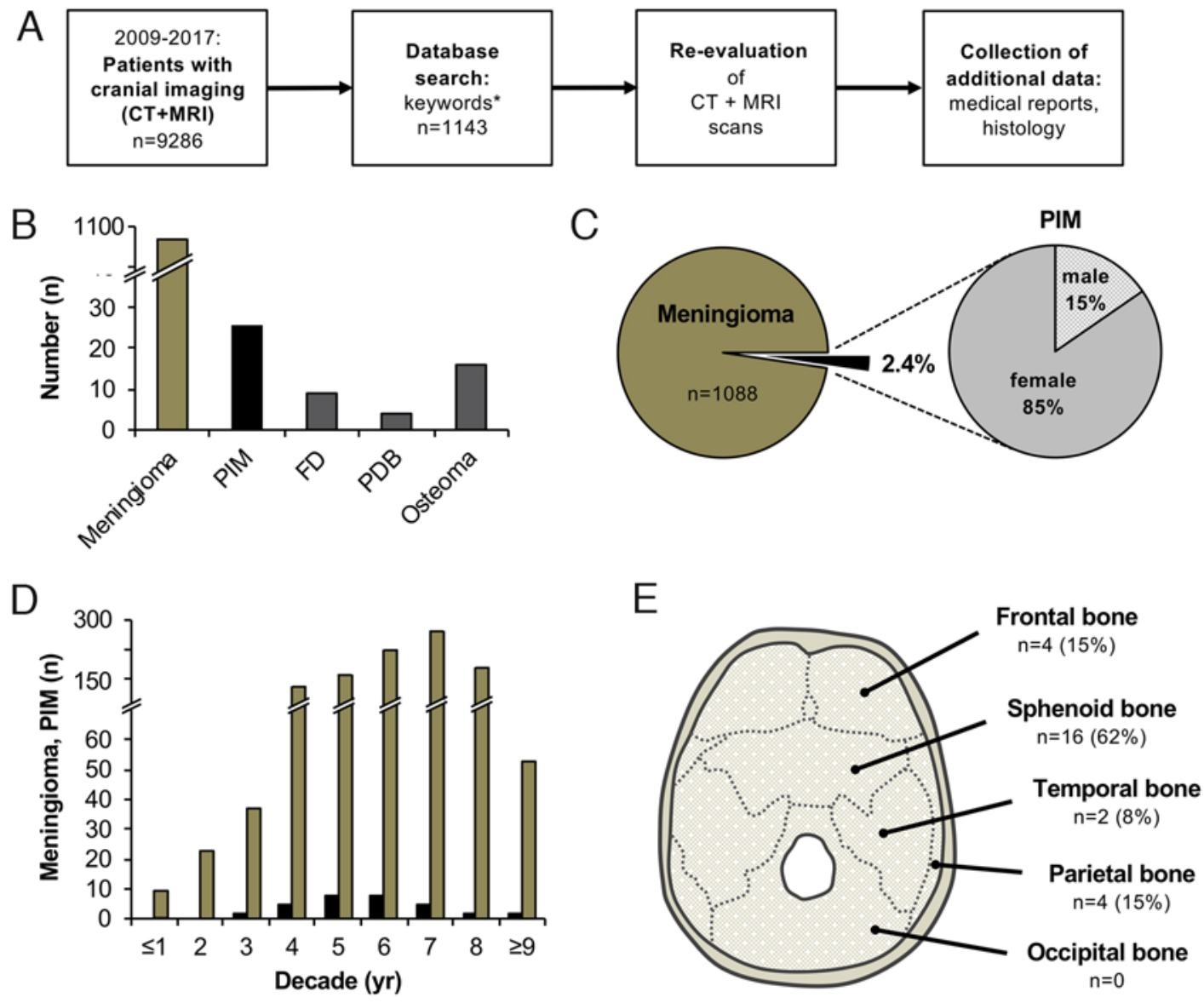

\begin{abstract}
FIG. 1. Epidemiological analysis. A: Flowchart of the methodological approach of the study. Keywords (asterisk) were as follows: "meningioma," "osseous," "intraosseous," "fibrous dysplasia," "paget," and "osteoma." B: The number of identified cases with meningioma, PIM, FD, PDB, and osteoma. C: The proportion and sex distribution of patients with PIMs. D: Age distribution of patients with meningiomas and PIMs. E: Localization and frequency of the PIMs within the skull bone. Figure is available in color online only.
\end{abstract}

clinical and histopathological points of view in order to improve the clinical management.

\section{Methods}

\section{Retrospective Database Analysis and Radiological Assessment}

In the period 2009-2017, a total of 9286 patients underwent radiological examination of the skull using both $\mathrm{CT}$ and MRI scans at our clinic. The local picture archive and communication system was searched for all cases of cranial meningioma and PIM, defined as a neoplastic lesion whose major portion is located within the bone, with no or only minimal dural involvement. Additionally, all cases of FD, PDB, and benign osteosclerotic lesions, such as osteoma, were identified. All selected cases of this preliminary study cohort were reviewed by a radiologist specialized in neuroradiology and by two orthopedists with extensive experience in rare bone diseases to confirm or to reject each initial diagnosis. For this purpose, clinical and pathological data, as well as the laboratory blood tests, of all patients were considered. In cases of incomplete or deficient data, which prevented the final clarification, respective cases were excluded. The resulting final study cohort formed the basis for the following analyses.

\section{Histological and Immunohistochemical Analysis}

Due to progressive pain and neurological deficits, microsurgical tumor resection of the osseous meningioma was performed in 16 cases. Since one case was operated on at an outside center, the data were not available for the following analysis. Thus, a total of 15 specimens were processed and underwent a standardized immunohistochemical analysis. The histological processing included fixation in formaldehyde (3.4\%) and dehydration in ascending concentrations of ethanol. Soft tissues were embedded in paraffin and stained with $\mathrm{H} \& \mathrm{E}$ and periodic acid-Schiff reaction. The bone specimens were prepared undecalcified in order to maintain their structure, histological characteristics, and especially the important infor- 
TABLE 1. Clinical characteristics of the study cohort

\begin{tabular}{|c|c|c|c|c|c|c|c|c|c|c|}
\hline \multirow[b]{2}{*}{$\begin{array}{l}\text { Case } \\
\text { No. }\end{array}$} & \multirow[b]{2}{*}{$\begin{array}{l}\text { Age } \\
\text { (yrs) }\end{array}$} & \multirow[b]{2}{*}{ Sex } & \multicolumn{3}{|c|}{ Radiology } & \multicolumn{2}{|c|}{ Clinical Symptoms } & \multicolumn{2}{|c|}{ Treatment } & \multirow[b]{2}{*}{$\begin{array}{c}\text { Simpson } \\
\text { Grade }\end{array}$} \\
\hline & & & $\begin{array}{l}\text { No. of } \\
\text { Tumors }\end{array}$ & Localization & Calcification & Preop & Postop & Surgery & RT & \\
\hline 1 & 71 & $\mathrm{~F}$ & 1 & Frontal & Yes & Lt hemiparesis & Symptom free & MTR, Al & No & 3 \\
\hline 2 & 56 & $\mathrm{~F}$ & 2 & $\begin{array}{l}\text { Frontal, sphe- } \\
\text { noidal }\end{array}$ & No & Exophthalmos & Symptom free & MTR & No & 3 \\
\hline 3 & 63 & $\mathrm{~F}$ & $1^{*}$ & Frontal & No & $\begin{array}{l}\text { Exophthalmos, bone } \\
\text { tumor }\end{array}$ & Symptom free & MTR & No & 3 \\
\hline 4 & 45 & $\mathrm{~F}$ & 1 & Parietal & Yes & $\mathrm{HA}$, bone tumor & Deceased (cerebral edema) & MTR, Al & No & 4 \\
\hline 5 & 69 & M & 1 & Sphenoidal & No & Visual loss, epilepsy & $\begin{array}{l}\text { Epilepsy, deceleration, } \\
\text { normalized vision }\end{array}$ & MTR & Yes & 4 \\
\hline 6 & 71 & $F$ & 1 & Sphenoidal & No & HA, exophthalmos & Symptom free & MTR & No & 4 \\
\hline 7 & 49 & $\mathrm{~F}$ & 1 & Sphenoidal & No & $\begin{array}{l}\text { Visual loss, exophthal- } \\
\text { mos }\end{array}$ & Symptom free & MTR & No & 4 \\
\hline 8 & 46 & $\mathrm{~F}$ & 1 & Sphenoidal & No & $\begin{array}{l}\text { Visual loss, exophthal- } \\
\text { mos }\end{array}$ & $\begin{array}{l}\text { Normalized vision \& } \\
\quad \text { exophthalmos, localized } \\
\text { pressure sensation }\end{array}$ & MTR & No & 4 \\
\hline 9 & 34 & $\mathrm{~F}$ & 1 & Sphenoidal & Yes & Exophthalmos & Epilepsy & MTR & No & 4 \\
\hline 10 & 68 & $\mathrm{~F}$ & 1 & Sphenoidal & No & $\begin{array}{l}\text { Exophthalmos, visual } \\
\text { loss, HA, lacrimation }\end{array}$ & $\begin{array}{l}\text { Vision, } \mathrm{HA} \text {, lacrimation nor- } \\
\text { malized; exophthalmos } \\
\text { diminishing }\end{array}$ & MTR & No & 4 \\
\hline 11 & 49 & M & 1 & Sphenoidal & No & Visual loss, lacrimation & $\begin{array}{l}\text { Consistently visual loss \& } \\
\text { lacrimation }\end{array}$ & MTR & Yes & 4 \\
\hline 12 & 53 & $\mathrm{~F}$ & 1 & Sphenoidal & No & Visual loss & Normalized vision & MTR & No & 4 \\
\hline 13 & 72 & $\mathrm{~F}$ & 2 & Sphenoidal & No & Visual loss & $\begin{array}{l}\text { Normalized vision, hydro- } \\
\text { cephalus, avolition }\end{array}$ & MTR & No & 4 \\
\hline 14 & 50 & $\mathrm{~F}$ & 1 & Temporal & Yes & $\mathrm{HA}$, bone tumor & Symptom free & MTR, Al & No & 1 \\
\hline 15 & 68 & $\mathrm{~F}$ & 1 & Temporal & No & Exophthalmos & Epilepsy & MTR & Yes & 4 \\
\hline 16 & 55 & M & 1 & Frontal & No & Bone tumor, pain & Symptom free & MTR & No & 4 \\
\hline 17 & 101 & $\mathrm{~F}$ & 1 & Parietal & Yes & NA & NA & CT & No & NA \\
\hline 18 & 64 & M & $1^{*}$ & Parietal & No & NA & NA & CT & No & NA \\
\hline 19 & 55 & $\mathrm{~F}$ & 1 & Parietal & Yes & $\mathrm{HA}$ & NA & CT & No & NA \\
\hline 20 & 55 & $\mathrm{~F}$ & 1 & Sphenoidal & No & NA & NA & CT & No & NA \\
\hline 21 & 86 & $\mathrm{~F}$ & 1 & Sphenoidal & Yes & NA & NA & CT & No & NA \\
\hline 22 & 66 & $\mathrm{~F}$ & 1 & Sphenoidal & Yes & NA & NA & CT & No & NA \\
\hline 23 & 66 & $F$ & 1 & Sphenoidal & Yes & NA & NA & CT & No & NA \\
\hline 24 & 57 & $\mathrm{~F}$ & 1 & Sphenoidal & Yes & NA & NA & CT & No & NA \\
\hline 25 & 41 & $\mathrm{~F}$ & 1 & Sphenoidal & No & NA & Epilepsy, depression & $\mathrm{CT}$ & No & NA \\
\hline 26 & 75 & $\mathrm{~F}$ & 1 & Sphenoidal & No & $\begin{array}{l}\text { Exophthalmos, visual } \\
\text { loss }\end{array}$ & No change & $\begin{array}{l}\text { CT, MTR rec- } \\
\text { ommended }\end{array}$ & No & NA \\
\hline
\end{tabular}

$\mathrm{Al}=3 \mathrm{D}$ artificial implant; $\mathrm{CT}=$ conservative therapy; $\mathrm{HA}=$ headache; $\mathrm{MTR}=$ microsurgical tumor resection; $\mathrm{NA}=$ not applicable; $\mathrm{RT}=$ radiation therapy.

* Meningiomatosis.

mation on the mineralization properties. This is essential for reliable analysis of the bone tissue. After embedding in methyl methacrylate, rotating grinders are used to reduce its thickness to $30 \mu \mathrm{m}$ or $5 \mu \mathrm{m}$, depending on the size of the specimen. The staining process with toluidine blue, trichrome Masson-Goldner, and von Kossa/van Gieson follows standard procedures. Our group has a long-term experience in processing and analyzing bone specimens in this manner. ${ }^{5,12,16,25}$

The immunohistochemical analysis comprised the epi- thelial membrane antigen as marker of meningiomas, and S100 and GFAP for differential diagnostics, as well as the progesterone receptor. Furthermore, the MIB1-antibody was used to determine the proliferative activity given as Ki-67 labeling index. All cases were classified using the WHO grading of meningiomas. ${ }^{17}$

\section{Bone Mineral Density Distribution}

The bone mineral density distribution of the hyperostotic bone tissue (case 20 [see Table 1]) was measured by 


\section{Primary Intraosseous Meningioma}

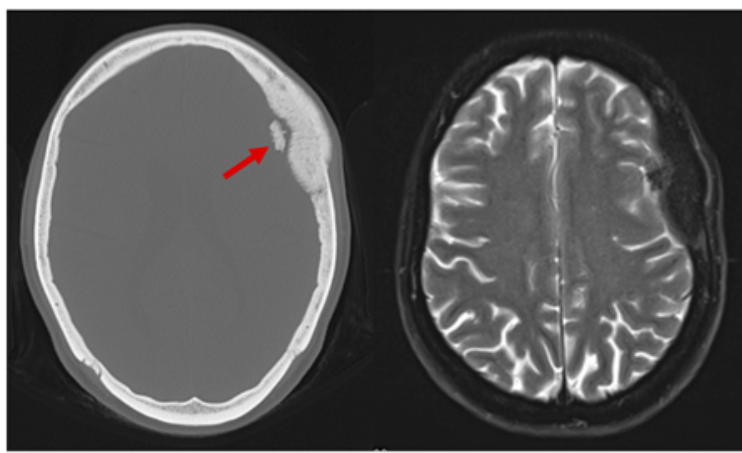

Fibrous Dysplasia

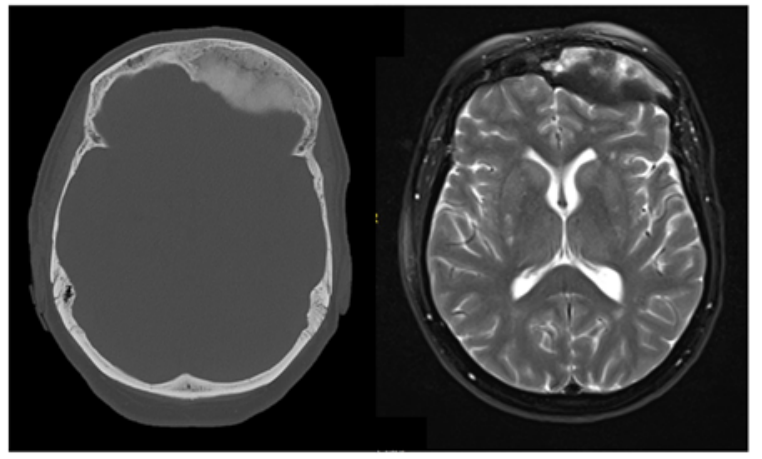

Metastasized Esthesioneuroblastoma

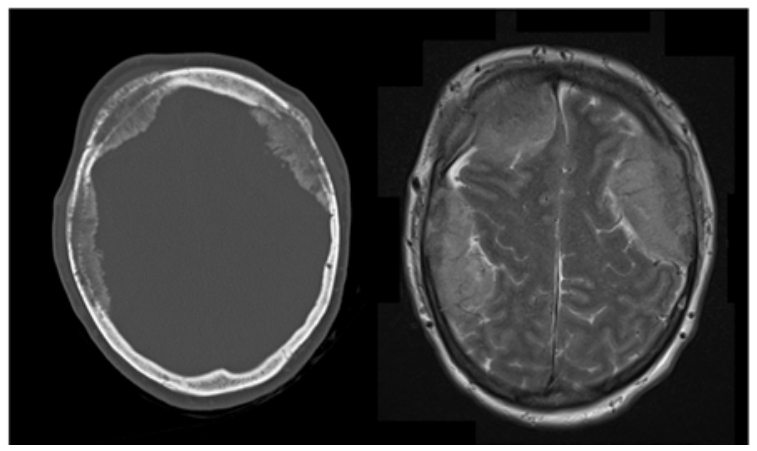

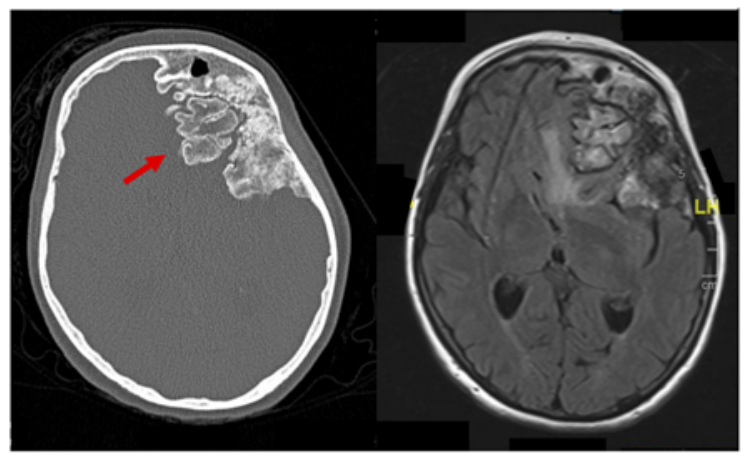

Paget's Disease

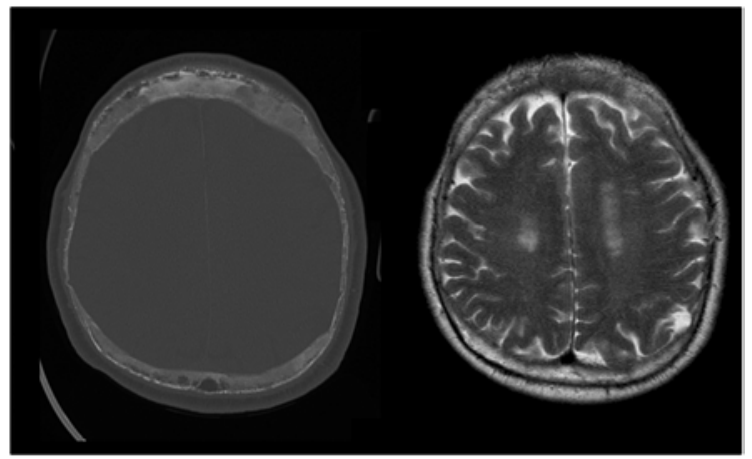

Hyperostosis frontalis interna

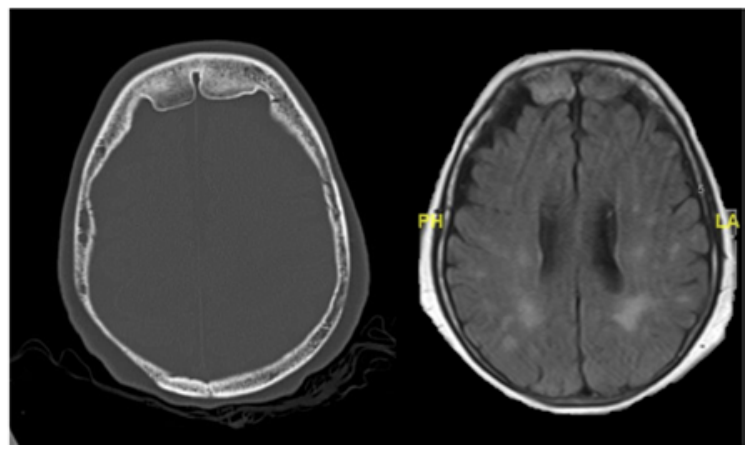

FIG. 2. Radiological presentation of PIMs and comparison with the most important differential diagnosis: each panel shows a CT scan (left) and the corresponding MRI scan (right); the primary intraosseous meningioma is given in a moderate (left) and an advanced (right) state in each panel, with characteristic calcifications (red arrows). Figure is available in color online only.

quantitative backscattered electron imaging (qBEI) (LEO 435 VP; LEO Electron Microscopy Ltd.) and compared to the normal surrounding trabeculae within the same specimen. The applied approach included the record of 4 images (magnification $\times 100$ ) and subsequent analysis using ImageJ (v.1.42, National Institutes of Health) following the previously published protocol. ${ }^{16} \mathrm{Ca}$ mean was determined by transformation of the grayscale values.

\section{Results}

Among the 1088 identified meningioma cases, PIM was diagnosed in 26 cases ( $85 \%$ female, $15 \%$ male), which corresponds to $2.4 \%$ of the meningiomas (Fig. 1A and B). From other cases with similar CT scan and MRI features,
9 cases were diagnosed with FD, 4 cases with PDB, and 16 cases with benign osteosclerotic lesions such as osteoma or hyperostosis frontalis interna (Fig. 1A). While the onset of meningiomas showed a peak in the 7 th decade, PIM occurred slightly earlier, peaking in the 6th decade (Fig. 1C). Regarding localization, the majority of PIMs were located within the sphenoid bone $(62 \%)$, while the other cases were in the frontal and parietal bones (15\% each) and in the temporal bone (8\%). The occipital bone was not affected in our study cohort (Fig. 1D).

Detailed radiological assessment showed that the vast majority of cases showed single manifestations of the PIM per case (Table 1$)$. Only $4(15.4 \%)$ of 26 cases had more than one manifestation. The patients in cases 2 and 13 had two separate PIMs of the sphenoid and frontal bone. The 

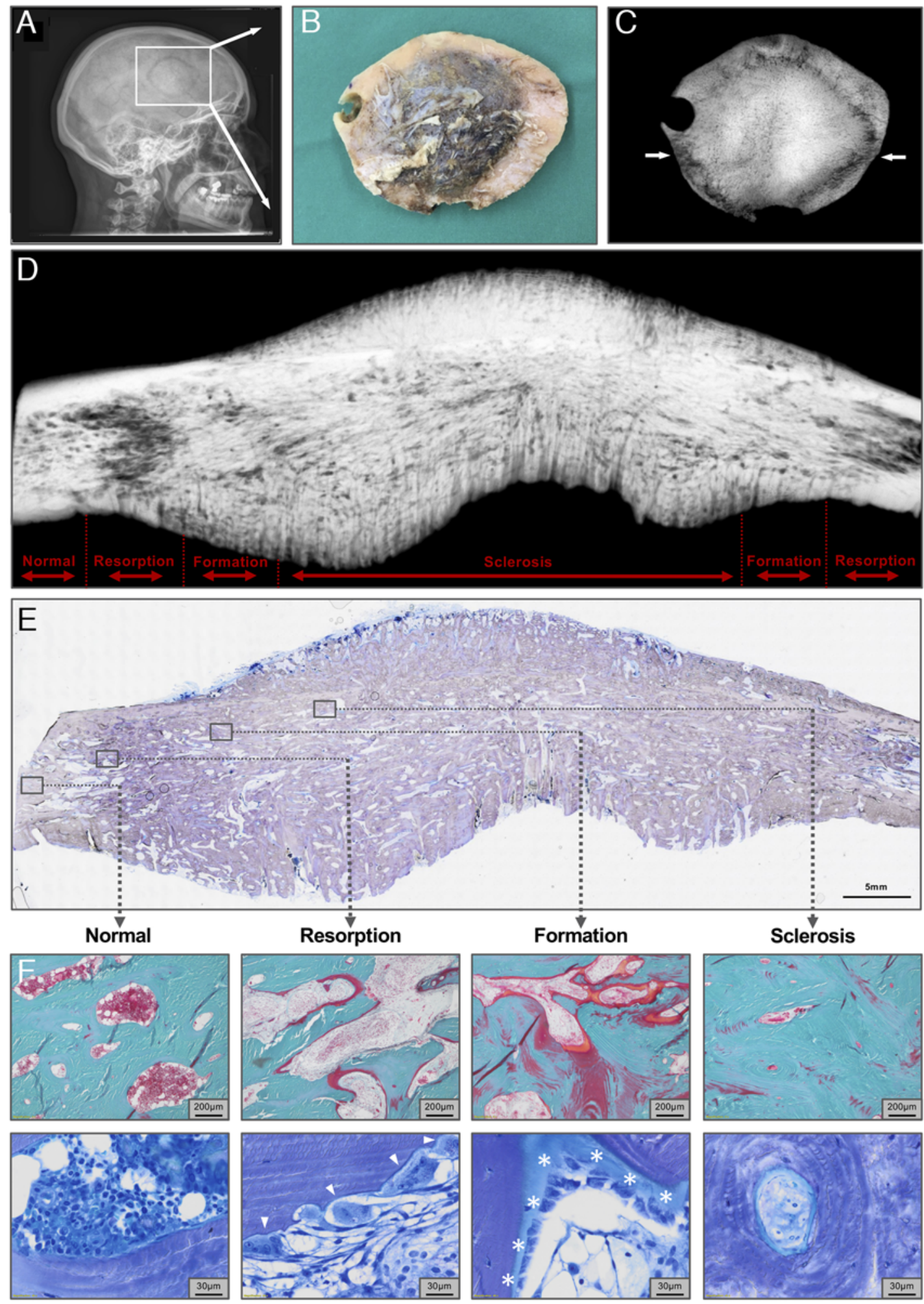

FIG. 3. Analysis of a complete cross section of a PIM. A: Lateral radiograph of the skull showing a circular lesion with a radiolucent border line (i.e., PIM). B: Photograph of the resected lesion. C: Contact radiograph of the resected skull bone. White arrows indicate the cut plane for subsequent processing. D: Contact radiograph of the complete cross section (maximum diameter $7.1 \mathrm{~cm}$ ) showing the dense hyperostosis with the characteristic frayed appearance and radiolucent regions, indicating the transition to the normal bone structure; red marks indicate the different stages of the bony changes connected to tumor growth (i.e., normal, resorption, formation, sclerosis). E: Corresponding overview image of the same section stained with toluidine blue (ground section, magnification $\times 5$, bar $5 \mathrm{~mm}$ ); while the existing skull bone is rather grayish colored, the newly formed bone tissue shows a purple appearance (inner and outer site of the skull bone as well as transition to the normal bone). F: High-magnification images (cut sections) stained with trichrome Masson-Goldner (upper row: magnification $\times 5$, bar $200 \mu \mathrm{m}$; changes in bone structure) and toluidine blue (lower row: magnification $\times 40$, bar $30 \mu \mathrm{m}$; corresponding cellular activity), representing the 4 stages in the development of the hyperostosis. Figure is available in color online only. 

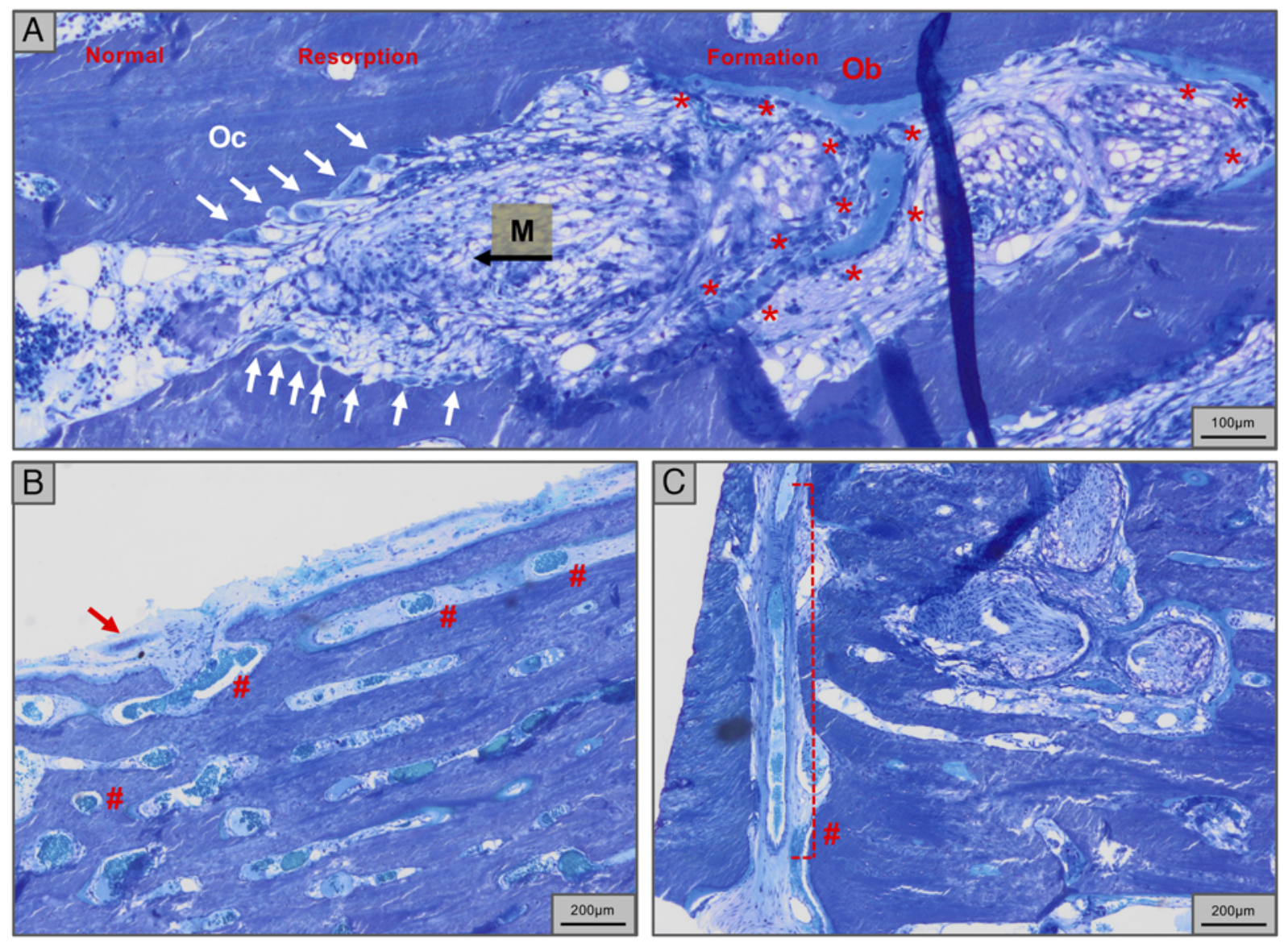

FIG. 4. Histological correlates of characteristic radiological findings in a ground section of the PIM. A: Overview of the expansion mechanism, including bone resorption by multiple osteoclasts (Oc; white arrows), invasion of tumor tissue ( $\mathrm{M}=$ meningioma; black arrow indicates the invasion direction), and consecutive deposition of osteoid by a large number of osteoblasts (Ob; red asterisks). B: Extracranial surface of the PIM with a layered structure of newly formed bone and a large number of blood vessels (red pound signs). The arrow indicates a newly formed bone island in the periphery, which is not connected to the adjacent bone layers in this 2D image. C: The intracranial surface contains long bone canals with blood vessels for the supply of the tumor tissue, resulting in the typical frayed appearance of the inner table in radiographs and CT scans. The pound sign indicates the bone canal containing a blood vessel, while the hashed line refers to the size of the vessel in this longitudinal cut section. Figure is available in color online only.

patients in cases 3 and 18 had multiple meningiomas and PIMs, resulting in a meningiomatosis. Isolated intracranial calcifications were visible in $58 \%$ of all cases (Table 1, Fig. 2). Clinical presentations ranged from asymptomatic (38\%) to significant neurological symptoms, including visual loss, exophthalmos, and headache. In $61 \%$ of the patients, the indication for a wide resection was given due to neurological failure and progressive growth. Eight patients were symptom free at the follow-up examinations after having undergone microsurgical tumor decompression. Another 4 patients showed at least an improvement in the clinical presentation compared to their preoperative states. However, 3 patients did not benefit from the operation. In fact, one patient died of postoperative complications (cerebral edema; Table 1). The mean follow-up period was 33 months. The Simpson grades of the surgically treated patients were 1 in one patient, 3 in three patients, and 4 in the majority of patients $(n=12)$. The other patients were treated conservatively and underwent follow-up examinations.
The detailed, multilevel analysis of the cranial specimen (case 14) revealed the characteristic periosteal bone apposition and the frayed appearance at the intracranial site of the bone on both the contact radiograph and the high-resolution overview image of the ground section (Fig. $3 \mathrm{~A}$ and $\mathrm{B}$ ). Using the histological cut sections of the same specimen, 4 stages of tumor growth (i.e., osseous reaction) could be identified: The surrounding area of normal bone tissue (1) underwent a massive increase in bone resorption (2) followed by ingrowth of tumor cells (Fig. 3C). The subsequently increased bone formation (3) with consecutive mineralization led to sclerosis of the calvarial bone, with only a minimum of soft-tissue volume (i.e., bone marrow) remaining (4). These morphological changes in the bone structure corresponded to the detection of vital bone marrow (1), large numbers of osteoclasts (2) and osteoblasts (3), as well as tumor tissue (i.e., loss of bone marrow; 4). An overview image of this growth pattern is given Fig. 4A. Additionally, the periosteal bone apposition is characterized by alternating layers of tumor tissue with hypervas- 
TABLE 2. Histological grading and immunohistochemical properties of the 15 patients with an indication for microsurgical tumor resection of the PIM

\begin{tabular}{cclccccc}
\hline Case No. & WHO Grade & Histological Diagnosis & EMA & S100 & GFAP & Progesterone Receptor & Ki-67 Index \\
\hline 1 & I & Meningotheliomatous & + & - & - & $30 \%$ & $<1 \%$ \\
\hline 2 & I & Transitional & + & - & - & $90 \%-100 \%$ & $4 \%$ \\
\hline 3 & I & Meningotheliomatous & + & - & - & Not available & Not available \\
\hline 4 & I & Meningotheliomatous & + & - & - & + & $1 \%$ \\
\hline 5 & II & Atypical & + & - & - & $90 \%-100 \%$ & $15 \%$ \\
\hline 7 & I & Not classifiable & + & - & - & + & $1 \%$ \\
\hline 8 & I & Meningotheliomatous & + & - & - & - & $2 \%$ \\
\hline 9 & I & Meningotheliomatous & + & - & - & $>80 \%$ & - \\
\hline 10 & I & Not classifiable & + & - & - & + & $<1 \%$ \\
\hline 11 & I & Meningotheliomatous & + & - & - & $80 \%-90 \%$ & Not available \\
\hline 12 & I & Meningotheliomatous & + & - & - & Not available & $\leq 5 \%$ \\
\hline 13 & I & Not classifiable & + & + & - & $80 \%$ & $<1 \%$ \\
\hline 14 & I & Meningotheliomatous & + & - & - & $30 \%-80 \%$ & $<3 \%$ \\
\hline 15 & I & Meningotheliomatous & + & + & - & - & $<\%$ \\
\hline
\end{tabular}

EMA = epithelial membrane antigen; GFAP = glial fibrillary acidic protein; NA = not available; + = positive; - = negative.

Histological data for case 16 was not available due to treatment at an external facility.

cularization and newly formed bone tissue (Fig. 4B). The frayed appearance of the intracranial bone site was found to be the result of bony canals containing large numbers of longitudinal blood vessels originating from the intracranial site to provide the blood supply for the growing tumor (Fig. 4C).

The histological analysis revealed WHO grade I with a meningotheliomatous differentiation in the majority of the cases $(93 \%$ and $91 \%$, respectively). Furthermore, two cases were classified as transitional (case 2) and atypical (case 5 ). Three cases could not be finally clarified. Detailed information regarding the immunohistochemical stains are summarized in Table 2.

The usage of polarized light microscopy showed the presence of original lamellar bone surrounded by large areas of newly formed woven trabeculae (Fig. 5A). The qBEI analysis confirmed this finding through a greater heterogeneity of the calcium distribution in the hyperostotic bone (CaWidth 4.4) when compared with the unaffected bone tissue (CaWidth 2.8; Fig. 5B). Additionally, a lower mean calcium (mean $22.7 \mathrm{wt} \%$ ) content was observed in the sclerotic bone tissue in comparison with the healthy tissue (mean 25.1).

\section{Discussion}

Our analysis revealed that only $2.4 \%$ of all included meningiomas were primary intraosseous meningiomas, making it indeed a rare entity. This small number is the cause for the limited knowledge and, in combination with an apparent rather unspecific radiographic appearance, may explain the challenging task of diagnosing this entity ${ }^{26}$ Previously reported percentages of primary extradural meningiomas were approximately $2 \% .^{9,13,19}$ Since PIMs are a subtype of these extradural tumors, our number should be considered slightly higher.
Concerning the sex ratio, different estimations for extradural meningiomas are available. While some studies reported an even male-to-female ratio ${ }^{19}$ or a slight female preponderance, ${ }^{9}$ we found a predominant occurrence in the female sex for PIMs. This tendency is in line with the sex ratio reported regarding intradural meningiomas. ${ }^{21,23}$ Regarding the onset of this rare entity, our data show a similar tendency in intradural and primary intraosseous meningiomas toward the second half of life; however, PIMs were diagnosed slightly earlier, with a peak in the 5 th and 6th decade. This difference may be explained by the predominant osseous manifestation. The resulting bulb is often palpable and leads to radiological examinations. ${ }^{7}$

According to our results, the majority of PIMs were located in the sphenoid bone (especially sphenoid ridge), explaining the frequent finding of exophthalmos and visual loss in our patients. In contrast to this, previous studies reported the predominant occurrence of PIMs in the calvarial bone, ${ }^{3,9,13,19}$ which is associated with a painless bulb or headache due to compression of the brain structures. A possible explanation for these differing findings may be seen in the anatomical crowding, making the radiological distinction of PIMs with minimal dural proliferation from intradural meningiomas with accompanying osseous reaction more difficult.

The diagnostic management in cases of suspected PIM includes both MRI and CT scans as well as laboratory blood tests. The key to diagnosis can be isolated calcifications or the irregularity of the inner or outer table, which can be seen best on CT scans. While MRI examinations only are not sufficient, they are still required in order to assess the surrounding soft tissue (e.g., dural involvement). Blood tests (e.g., alkaline phosphatase) can rule out an important differential diagnosis such as PDB. ${ }^{24}$ Although we cannot exclude alkaline phosphatase eleva- 

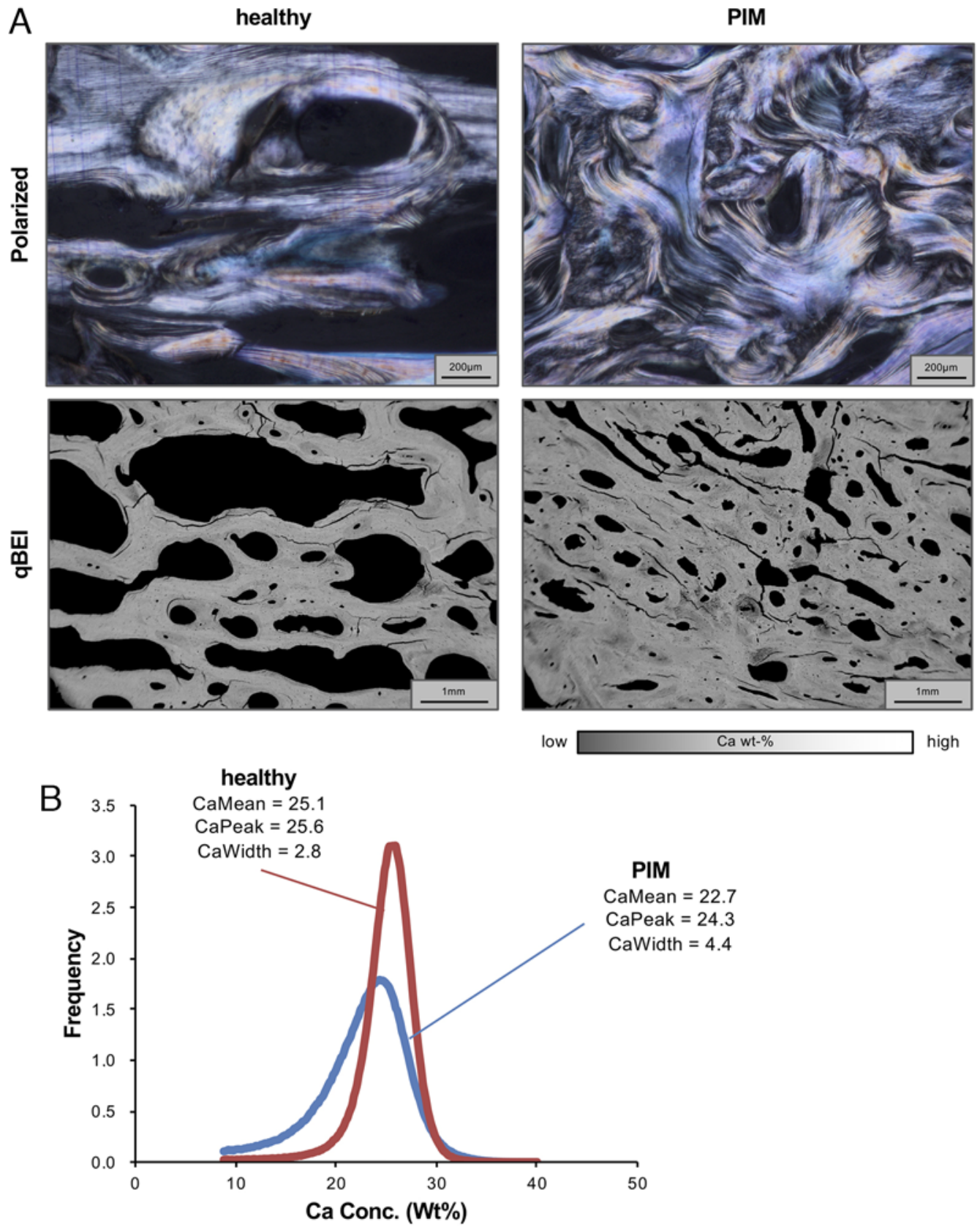

FIG. 5. In-depth analysis of the bone structure (hyperostosis vs normal bone). A: Polarized light microscopy (bar $200 \mu \mathrm{m}$ ) reveals lamellar bone tissue in the normal control specimen; the PIM specimen shows a dense, irregular mixture of existing lamellar bone and woven bone, which can be also partially observed in the qBEl images (bar $1 \mathrm{~mm}$ ). B: qBEI data showing a lower mean and inhomogeneously distributed calcium content in the hyperostosis specimen when compared with the normal control. $\mathrm{Ca}=\mathrm{calcium}$; conc. $=$ concentration. Figure is available in color online only.

tions in PIM, we did not observe this in our study. In rare cases of missing hyperostotic or lytic bone changes, the additional scintigraphy can be helpful: the persistent increase in scintigraphic uptake may indicate the presence of a PIM..$^{15,29}$

It has previously been discussed whether hyperostosis is a secondary phenomenon and may not constitute osseous tumor invasion..$^{22}$ In this morphological study, comparing radiographs and histological specimens, the authors could show that hyperostotic changes were indeed associated with direct invasion of tumor tissue into the bone. ${ }^{22}$ Our histological findings support these results and provide additional insights into the pathological mechanism of tumor growth within the bone: first, the growth of the tumor tissue is accompanied by a massive increase in the osteoclast activity with resorption of the normal trabeculae. As a result, a thinning of the bone (best seen in calvarial locations) develops at the transition of the invaded to 
the normal bone, which can be seen on plain radiographs as a radiolucent borderline. Subsequently, the tumor cells invade this area, accompanied by an increase in osteoblast activity with deposition of osteoid. The analysis of the inner table of the skull bone showed a frayed and irregular appearance, which was previously described in calvarial PIM. ${ }^{20}$ According to our histological results, this configuration results from the ingrowth of large vessels. On the other hand, the irregular appearance of the outer table is caused by a layered structure of tumor tissue and irregular bone formation and a dense network of blood vessels. A previous work demonstrated that extracranial vessels, such as the external carotid artery, can provide additional blood supply for the PIM. ${ }^{6}$ Furthermore, the hyperostotic changes at the inner and outer table resemble periosteal reactions, which are known from inflammatory or traumatic events. ${ }^{4}$ Thus, the hyperostosis may be the consequence of the changes in the bone structure induced by the tumor growth. ${ }^{10}$

These observations have two major consequences: first, they support the theory of hyperostotic changes being directly connected to tumor invasion, which elucidates the need for wide total resections, including the radiolucent borderlines; second, the bone turnover (i.e., osteoclast and osteoblast activity) may play a central role in the tumor growth. Therefore, antiresorptive drugs such as bisphosphonates should be considered as adjuvant treatment concepts. A previous case report observed a reduction in the clinical symptoms as well as in the scintigraphic uptake of the skull region after the administration of etidronate $200 \mathrm{mg} /$ day for 6 months. ${ }^{14}$ Particularly those patients who are not amenable to total resection procedures may benefit from such approaches. In such cases, other measures, such as radiotherapy, chemotherapy, and hormone therapy, are also available. ${ }^{2,11}$

As a limitation, this study cohort includes only patients with hyperostotic bone reactions. Groups with mixed or lytic changes may have altered properties, especially in terms of epidemiological data (e.g., age, sex ratio) and the underlying cellular mechanism. Furthermore, the diagnosis in the other 11 hyperostotic PIM cases, which were not surgically treated, was not confirmed histologically. Additionally, a comparison with standard meningiomas was not done. Finally, the histological specimens are static samples, which do not allow for conclusions of dynamic processes (e.g., tumor growth). However, this unique analysis of a complete cross section of the PIM may show the different stages (i.e., mechanism) of the bony changes in PIM.

\section{Conclusions}

We found similar epidemiological data in both intradural meningiomas and PIMs, with a clear predominance in women. In particular, the consideration of calcifications may lead to the correct diagnosis on the obligatory CT or MRI scans. A sequence of cellular activity with bone resorption, tumor invasion, and bone formation may explain the development of the characteristic hyperostotic changes and provides new approaches for alternative treatment options.

\section{References}

1. Agrawal V, Ludwig N, Agrawal A, Bulsara KR: Intraosseous intracranial meningioma. AJNR Am J Neuroradiol 28:314315, 2007

2. Apra C, Peyre M, Kalamarides M: Current treatment options for meningioma. Expert Rev Neurother 18:241-249, 2018

3. Arana E, Diaz C, Latorre FF, Menor F, Revert A, Beltrán A, et al: Primary intraosseous meningiomas. Acta Radiol 37:937-942, 1996

4. Bisseret D, Kaci R, Lafage-Proust MH, Alison M, ParlierCuau C, Laredo JD, et al: Periosteum: characteristic imaging findings with emphasis on radiologic-pathologic comparisons. Skeletal Radiol 44:321-338, 2015

5. Butscheidt S, Moritz M, Gehrke T, Püschel K, Amling M, Hahn M, et al: Incorporation and remodeling of structural allografts in acetabular reconstruction: multiscale, micromorphological analysis of 13 pelvic explants. J Bone Joint Surg Am 100:1406-1415, 2018

6. Changhong L, Naiyin C, Yuehuan G, Lianzhong Z: Primary intraosseous meningiomas of the skull. Clin Radiol 52:546549, 1997

7. Chen TC: Primary intraosseous meningioma. Neurosurg Clin N Am 27:189-193, 2016

8. Daffner RH, Yakulis R, Maroon JC: Intraosseous meningioma. Skeletal Radiol 27:108-111, 1998

9. Elder JB, Atkinson R, Zee CS, Chen TC: Primary intraosseous meningioma. Neurosurg Focus 23(4):E13, 2007

10. Fratzl-Zelman N, Roschger P, Kang H, Jha S, Roschger A, Blouin S, et al: Melorheostotic bone lesions caused by somatic mutations in MAP2K1 have deteriorated microarchitecture and periosteal reaction. J Bone Miner Res [epub ahead of print], 2019

11. Gupta S, Bi WL, Dunn IF: Medical management of meningioma in the era of precision medicine. Neurosurg Focus 44(4): $\mathrm{E} 3,2018$

12. Hahn M, Vogel M, Delling G: Undecalcified preparation of bone tissue: report of technical experience and development of new methods. Virchows Arch A Pathol Anat Histopathol 418:1-7, 1991

13. Ilica AT, Mossa-Basha M, Zan E, Vikani A, Pillai JJ, Gujar $\mathrm{S}$, et al: Cranial intraosseous meningioma: spectrum of neuroimaging findings with respect to histopathological grades in 65 patients. Clin Imaging 38:599-604, 2014

14. Inagaki K, Otsuka F, Matsui T, Ogura T, Makino H: Effect of etidronate on intraosseous meningioma. Endocr J 51:389390, 2004

15. Kanmaz B, Weissman DE, Akansel G, Kitapci M, Collier $\mathrm{BD}$ : Intraosseous meningioma: appearance on bone scintigraphy over five years. J Nucl Med 34:961-962, 1993

16. Koehne T, Vettorazzi E, Küsters N, Lüneburg R, Kahl-Nieke $\mathrm{B}$, Püschel K, et al: Trends in trabecular architecture and bone mineral density distribution in 152 individuals aged 30-90 years. Bone 66:31-38, 2014

17. Kresak JL, Yachnis AT: WHO grading of meningiomas. PathologyOutlines.com. (http://www.pathologyoutlines. com/topic/cnstumorwhomeningioma.html) [Accessed April 25, 2019]

18. Kwon SY, Shin HS, Kim TH, Kim HJ: Primary intraosseous osteolytic meningioma of the skull mimicking scalp mass: a case report and review of literature. Brain Tumor Res Treat 3:151-155, 2015

19. Lang FF, Macdonald OK, Fuller GN, DeMonte F: Primary extradural meningiomas: a report on nine cases and review of the literature from the era of computerized tomography scanning. J Neurosurg 93:940-950, 2000

20. Matschke J, Addo J, Bernreuther C, Zustin J: Osseous changes in meningioma en plaque. Anticancer Res 31:591-596, 2011

21. Ostrom QT, Gittleman H, Farah P, Ondracek A, Chen Y, Wo- 
linsky Y, et al: CBTRUS statistical report: Primary brain and central nervous system tumors diagnosed in the United States in 2006-2010. Neuro Oncol 15 (Suppl 2):ii1-ii56, 2013

22. Pieper DR, Al-Mefty O, Hanada Y, Buechner D: Hyperostosis associated with meningioma of the cranial base: secondary changes or tumor invasion. Neurosurgery 44:742-747, 1999

23. Rohringer M, Sutherland GR, Louw DF, Sima AA: Incidence and clinicopathological features of meningioma. J Neurosurg 71:665-672, 1989

24. Rolvien T, Butscheidt S, Zustin J, Amling M: Skeletal dissemination in Paget's disease of the spine. Eur Spine J 27 (Suppl 3):453-457, 2018

25. Rolvien T, Schmidt FN, Milovanovic P, Jähn K, Riedel C, Butscheidt S, et al: Early bone tissue aging in human auditory ossicles is accompanied by excessive hypermineralization, osteocyte death and micropetrosis. Sci Rep 8:1920, 2018

26. Tokgoz N, Oner YA, Kaymaz M, Ucar M, Yilmaz G, Tali TE: Primary intraosseous meningioma: CT and MRI appearance. AJNR Am J Neuroradiol 26:2053-2056, 2005

27. Vlychou M, Inagaki Y, Stacey R, Athanasou NA: Primary intraosseous meningioma: an osteosclerotic bone tumour mimicking malignancy. Clin Sarcoma Res 6:14, 2016

28. Wang Y, Wang Y, Xu H, Li Y: Primary intraosseous lymphoplasmacyte-rich meningioma: a case report. World Neurosurg 109:291-293, 2018
29. Zaritzky D, Cowan RJ: Intraosseous meningioma: an unusual radionuclide presentation. J Nucl Med 18:943-944, 1977

\section{Disclosures}

The authors report no conflict of interest concerning the materials or methods used in this study or the findings specified in this paper.

\section{Author Contributions}

Conception and design: Amling, Butscheidt, Martens. Acquisition of data: Butscheidt, Rolvien, Hubert, Martens. Analysis and interpretation of data: Amling, Butscheidt, Ernst, Rolvien, Zustin, Martens. Drafting the article: Butscheidt, Ernst, Rolvien, Hubert. Critically revising the article: Amling, Butscheidt, Ernst, Hubert, Zustin, Martens. Reviewed submitted version of manuscript: all authors. Statistical analysis: Butscheidt. Administrative/technical/ material support: Amling, Ernst, Zustin, Martens. Study supervision: Amling, Martens.

\section{Correspondence}

Michael Amling: University Medical Center Hamburg-Eppendorf, Hamburg, Germany. amling@uke.de. 